\title{
The efficacy of a training program for social skills in reducing addictive Internet behaviors among Palestinian university students
}

\author{
Saida Affouneh', Fayez Azez Mahamid ${ }^{1 *}$ (D, Denise Ziya Berte ${ }^{2}$, Ali Z. Shaqour ${ }^{1}$ and Marouf Shayeb ${ }^{1}$
}

\begin{abstract}
Background: For youth in geopolitically at-risk environments, such as Palestine, the issues related to Internet overuse and addictions are complex. Youth residing in the occupied territories of Palestine as in other highly militarized zones have high levels of environmental stressors (e.g., militarization, poverty, lack of employment opportunities, cultural pressures, etc.) and few chemical or social outlets such as alcohol, intoxicants, and leisure activities. As such, the easily accessible and unrestricted opportunities for stress-reducing social contact of social media can lead easily to excessive and maladaptive Internet use. Therefore, interventions directly aimed at awareness and education on how to manage Internet use are critical for addressing these issues in high risk populations.
\end{abstract}

Aims: The purpose of the current study was to test the efficacy of a time-limited group training program aimed at improving social skills and reducing addictive Internet behaviors among university students.

Methods and results: The sample consisted of 30 university students who self-reported high scores on an Internet addiction scale. Participants were randomly assigned to either wait list or treatment group (15 in each condition). Results demonstrated that using a social skills training program over an 8-week period improved the level of social skills and reduced addictive Internet behaviors significantly in those who experienced the intervention when compared with a wait list control group.

Conclusions: These findings support the implementation of time limited training programs targeting social skills and addictive patterns of Internet use with university students identified with high levels of Internet addictive behaviors.

Keywords: Social skills, Addictive Internet behaviors, University students, Palestine

\section{Introduction}

The use of social media has grown exponentially in the past decade to the extent of engaging close to one third of the world's population as of January 2017 (Hawi and Samaha 2017). This phenomenon is facilitated by numerous active social media sites

\footnotetext{
* Correspondence: Mahamid@najah.edu

${ }^{1}$ An-Najah National University, Nablus, Palestine

Full list of author information is available at the end of the article
}

including Facebook, Instagram, Twitter, and LinkedIn. As of December 2020, on average, Facebook had more than 2.6 billion monthly active users (Facebook 2020); Instagram reports more than 1 billion monthly active users, and 500 million individuals shared stories daily (Instagram 2020); Twitter had 330 million monthly active users, and 500 million tweets sent per day (Twitter 2020); and LinkedIn had more than 675 million active users (LinkedIn 2018). In the arena of gender differences, slightly more women $(68 \%$ of the 
total population) use social media compared to men (62\% of the total population), but women are higher utilizers who on average spend $46 \mathrm{~min}$ per day on social media compared to $31 \mathrm{~min}$ by men (Blachnie et al. 2017).

Social media addiction is defined as the compulsive use of social media sites that manifests itself in behavioral addiction symptoms. The symptoms include tolerance (increased use over time); conflict of use with physical, social, vocational, or academic obligations; withdrawal (feeling of distress when unable to use); relapse (inability to make decisions about use time or decide when you should stop using); and mood modification (euphoria or comfort with use, irritability or distress without use) (Chiu 2014).

Individuals, especially youth, who have not yet fully developed the skills to manage situational stressors, may both loose critical exposure to non-virtual social and academic experiences while spending extensive use time and also may have longer-term effects of over-use of the Internet due to having limited welltrained alternative problem-solving responses to environmental stressors later in life. Youth with addictive behavior patterns frequently do not develop healthy, positive, and action-based coping skills, when faced with external stressors. People with addictive habits generally have reduced local social support systems, despite having extensive addiction-related contacts (Mahamid and Berte 2019).

The risk of potential negative effect of excessive social media use appears to be increased for individuals with restricted resources (limited social outlets, challenges with mobility, sparse recreational activities, etc.) and developmental vulnerability with some age groups at higher risks, particularly young adults (Mahamid and Berte 2020).

Some studies have examined the relationship between social skills and the use of social media sites and found that people with lower levels of social skills tend to have higher frequency and intensity use of social media sites with the intended goal of enhancing their self-image and self-esteem. However, the desired effect is not always achieved as social media use is also often found to be linked to depression and a reduction of life satisfaction (Chang et al. 2017; Espada et al. 2014; Greco et al. 2009; Mclaughlin and Whitty 2007).

A longitudinal study of Facebook users showed that social skills were a factor that moderated the relation between Facebook use and self-esteem, whereas users with low levels of social skills and or self-esteem benefited from additional Facebook use to increase their social capital. Based on this social compensation hypothesis, people with low social skills, with low life satisfaction, and who have few offline contacts compensate by using
Facebook to gain more friends and more perceived popularity (Lampe et al. 2008).

When we engage in face-to-face communication social information is conveyed by vocal and visual cues in the context of the situation. Non-verbal communication is an important part of a message and it includes facial expressions, eye contact, tone of voice as well as posture, space between individuals, etc. (Bambaeeroo and Shokpour 2017).

Understanding the non-verbal aspects of communication is crucial because in social situations we need to modify our message or behavior in response to the reactions of others. Our ability to identify, interpret, and appropriately react to emotional cues is associated with personal, social, and academic success (Knapp, Hall, \& Horgan 2013). Moreover, youth who understand emotional cues in social settings develop superior social skills and attain more positive peer relationships (Blakemore 2008). These non-verbal, affective cues are much more evident communicating in person vs. digitally (Sherman et al. 2013). Youth using digital communication extensively may not have the face-to-face experiences necessary for them to develop and master important social skills (Giedd 2012).

The literature features a number of studies that showed that Internet addictions, including excessive use of the Internet and social networking sites, correlate positively with stress, anxiety, and lack of social skills as well as negative associations with academic performance, all of which negatively affect perceived self-efficacy and self-esteem (Hawi and Samaha 2016; Kabasakal 2015; Kuss et al. 2014; Lepp et al. 2014).

Wolfing et al. (2011) found that Internet addiction was related to high levels of dysfunctional (maladaptive) coping strategies. These findings are supported by Sriwilai and Charoensukmongkol (2015) who indicated that an avoidant coping style was related to generalize problematic Internet use.

Espada et al. (2014) found that adolescents with high scores in problematic use of the Internet present concordant higher scores in social anxiety and social skills difficulties. Furthermore, adolescents with Internet addictive behaviors showed a positively significant association both with the degree of social anxiety and with lack of assertiveness. On the other hand, the interpersonal addiction did more with social anxiety.

Blachnie et al. (2017) tested the association between Facebook addiction, social skills, and self-esteem among 381 Polish Facebook users who use daily more than 7 h; results indicated a negative relationship between Facebook addiction, social skills, and self-esteem among participants. These results are in agreement with Satici and Uysal (2015) who tested the relationship between problematic Facebook use and life satisfaction among 
Turkish undergraduate students. Results showed a negative relationship between problematic Internet use and life satisfaction.

The importance of social interaction in Internet addiction treatment includes increasing frequency and quality of social contacts, which will enhance social skills as well as the increasing frequency, and quality of live contacts (Khazaei, Khazaei, \& Ghanbari, 2017). Yao and Zhong (2014) presume that an increase in the frequency and quality of face-to-face social contacts can reduce Internet addiction.

The Social Stress Model of addiction proposes that adolescents engaged in addictive behaviors use it as a strategy to cope with stressors in different aspects of their life. It further proposes that improving social skills and support are critical in helping adolescents more effectively manage the stressors that arise during this period. Supporting the model, empirical evidence suggests that a higher level of social skills and support are associated with lower levels of addictive behaviors (Lau et al. 2018).

According to a social-cognitive theory of generalized problematic Internet use, improving social skills among individuals with Internet addictive behaviors should be related negatively with addictive behaviors. Individuals with Internet addiction frequently experience unpleasant social interactions in real life and appraise themselves as socially incompetent in face-to-face interactions. Yet, these individuals tend to perceive themselves as having greater confidence and efficacy in online interactions. Hence, individuals with Internet addiction prefer online interactions while avoiding face to-face communication (Cheng et al. 2017).

Generally, social skills training programs to reduce addictive behaviors include three major components: one component is very similar to the prevention approach that focuses on helping individuals resist social influences to use Internet intensively. The two other components focus on competence enhancement with an emphasis on teaching self-management skills and general social skills (Botvin and Griffin 2005).

Social skills training which focuses on teaching necessary skills should be an effective strategy for adolescents who suffer from Internet addiction. Communication skills would increase and improve problem-solving skills/outcomes, which should significantly decrease Internet addiction. Generally, it is held that promoting communication skills is effective in psycho-cognitive and mental health status. These skills may help the individual in dealing effectively with life stressors instead of engaging in addictive behaviors (Spencer 2006).

Social skills help individuals in starting conversations, listening actively and openly expressing thoughts and emotions. These skills are important in helping individuals to decrease negative feelings and social tensions and constructively/successfully solve problems which lead to preventing the emergence of negative and unconstructive behaviors such as those in Internet addictive (Abolfathi et al. 2014).

Cognitive behavioral therapy (CBT) has been shown to be an effective treatment for improving social skills as a model to deal with compulsive disorders such as Internet addiction (Cully and Teten 2008). CBT is a familiar treatment based on the premise that thoughts determine feelings. Individual are taught to monitor their thoughts and identify those that trigger addictive feelings and actions while learning new coping skills and ways to prevent a return to depression, anxiety, or addiction-based behavior patterns ( $\mathrm{Du}$ et al. 2010). The early stage of CBT is behavioral, focusing on specific behaviors and situations that maintain the excessive Internet use. As therapy progresses, there is more of a focus on the cognitive assumptions and distortions that have developed and the effects of these on behavior. This treatment involves assessment of the type of distortion, problemsolving skills and coping strategies training, modeling in therapy, support groups, and keeping thought journals (Zarb 2007).

Recent studies have yielded the efficacy of training and therapeutic programs in reducing Internet addiction and improving social skills. For example, Fang et al. (2015) examined the efficacy of multi-generational group therapy to reduce Internet addiction and improve social skills among adolescents. Results indicated that adolescents and parents who received the training program were significantly improved compared to the control group.

These outcomes are consistent with those reported by Halasy et al. (2017) who tested the efficacy of a cognitive-behavioral group intervention aimed at preventing extensive Internet use in adolescents. Outcomes showed significant reduction of Internet use disorder and comorbid symptoms as well as the promotion of problem solving, cognitive restructuring, and emotion regulation skills.

These results are supported by Yang and Kim (2018) who explored the efficacy of a self-regulatory program on self-control, self-efficacy, Internet addiction, and time spent on the Internet among 79 middle school students in South Korea. Results showed that self-control and self-efficacy significantly increased and Internet addiction and time spent on the Internet significantly decreased in the intervention group compared with the control group.

In 2014, Palestine was ranked the eighth on the list of Arab countries related to the percentage of the population using social networking sites by more than 33\% (Concepts). The number of Palestinian using Facebook is currently $1,520,000$ of 4.1 million Palestinians in the 
West Bank and Gaza Strip (Mourtada and Salem 2015). The situation of Palestinian university students in the occupied territories of Palestine is fraught with environmental stressors (militarization, poverty, lack of employment opportunities, cultural pressures, etc.) and few positive social outlets due to the restrictions on movement between communities, a lack of recreational facilities, and cultural standards of gender separation. In this situation, it is likely that a vulnerability to the easily accessible and unrestricted social networks of social media could lead easily to excessive and maladaptive use in the face of heightened stressors and few alternative avenues for socialization (Mahamid and Berte 2019). Research suggests that Palestinian college students' greater accessibility and usage of the Internet may increase their vulnerability to Internet abuse (Mahamid and Berte 2020).

According to a survey conducted at An-Najah National University, more than $47 \%$ of students are engaging in addictive patterns of use related to the Internet, with social communications as their first priority, only a minority of students report to Internet use for academic tasks or business opportunities (Berte et al. 2021).

In response to this disconcerting finding, the current study was designed to test the efficacy of a training program to enhance social skills and reduce addictive Internet behaviors of Palestinian university students who are at risk more than any category of Palestinian population to develop Internet addiction in order to decrease their social media dependence, and direct them to the positive Internet use for learning and business.

The current study hypothesized that (1) there would be a negative association between Internet addiction and social skills among Palestinian university students and (2) the training program based on social skills would decrease Internet addiction symptoms among university students.

\section{Methodology \\ Participants}

The sample of this study was selected from An-Najah University students after applying the Internet Addiction Test (IAT) created by Young (2012) and Social Skills Scale created by Moran et al. (2015). The two scales were uploaded to the Website of An-Najah National University, students who were interested in participating in this research were asked to voluntarily answer the questions of scales. Students whose scores ranged at risk included 60 students, 30 of which agreed to participate in the study. Inclusion in the study required participants to be (1) Palestinian university students, (2) native Arabic speakers, (3) residing in the West Bank of Palestine, and (4) engaged in excessive Internet usage.

The total number of the study sample was 30 participants representing 14 males and 16 females. A geographical representation of the participants showed that $57.0 \%$ of participants were from cities. Thirty-three percent were from villages, and $10.0 \%$ were from Palestinian internally displaced people's camps. Selected students were randomly assigned into two groups; 15 participants were exposed to the training program, and another 15 participants considered as a control group did not receive any intervention but instead placed on a wait list for treatment. The performance of the two groups on addictive behaviors and social skills was measured before and after the intervention.

\section{Study instruments \\ Internet Addiction Test (IAT)}

The IAT created by Young (2012) was used to measure the variable of level of addictive behaviors. The IAT is a reliable and valid measure of addictive use of Internet, developed by Kimberly Young. It consists of 20 items that measure mild, moderate, and severe levels of Internet addiction. Mahamid and Berte (2019) validated the scale in Palestinian context by using construct and content validity; the scale ended up with 19 items to test Internet addiction. In this study, the reliability coefficients of the scale was found to be $\alpha=.90$.

\section{Social Skills Questionnaire (SSQ-U)}

The SSQ-U created by Moran et al. (2015) was used to test the variable of social skills. The SSQ-U is a reliable and valid instrument to test social skills among university students, including social skills for academic and workplace settings, refusal assertiveness, commendatory assertiveness, and social skills for affective and conversational skills. After translating the questionnaire to Arabic, a committee of experts in psychology reviewed the items of the scale for content validity and comprehensiveness. In this study, the reliability coefficients of the scale was found to be $\alpha=.93$.

\section{Training program}

The researchers developed the training program based on the theoretical and applied literature in this filed (Balci and Uysal 2018; Celik 2016; Champion et al. 2016; Fang et al. 2011; Fang et al. 2015; Halasy et al. 2017; Yang and Kim 2018; Liu and Wang 2017). The training program consisted of 8 sessions, one session per week; each session lasted for $90 \mathrm{~min}$, each of which included aims, exercises, and a home work to improve social skills and reduce addictive behaviors among participants. Sessions were held in a group training room at the Faculty of Educational Sciences at An-Najah National University. Group members attended all training sessions, which were conducted by one of the authors who is licensed trainer of psychologists. Table 1 shows the training program. 
Table 1 Training-program sessions to increase social skills and reduce addictive behaviors

\begin{tabular}{|c|c|}
\hline Sessions & Strategies \\
\hline $\begin{array}{l}\text { 1st session } \\
\text { Relationship building }\end{array}$ & $\begin{array}{l}\text { Group introductions } \\
\text { Inform members about the process } \\
\text { Share expectations with the group } \\
\text { Determine personal aims related to the process } \\
\text { Determine rules for the group } \\
\text { Assessment and summary of session }\end{array}$ \\
\hline $\begin{array}{l}\text { 2nd session } \\
\text { Internet use and social skills }\end{array}$ & $\begin{array}{l}\text { Become aware of the Internet addictive behaviors } \\
\text { Observe negative aspects of long-term Internet use } \\
\text { Observe the correlation between excessive Internet use and lack of social skills } \\
\text { Use the Internet consciously } \\
\text { Assessment and summary of session }\end{array}$ \\
\hline $\begin{array}{l}\text { 3rd session } \\
\text { Self-discipline }\end{array}$ & $\begin{array}{l}\text { Learn strategies for self-control to deal with addictive behaviors } \\
\text { Apply these strategies } \\
\text { Determine functional aims } \\
\text { Assessment and summary of session }\end{array}$ \\
\hline $\begin{array}{l}\text { 4th session } \\
\text { Effective problem-solving } \\
\text { strategies }\end{array}$ & $\begin{array}{l}\text { Learn problem-solving strategies to deal with psychological, social, and emotional problems to prevent addictive } \\
\text { behaviors } \\
\text { Apply these strategies } \\
\text { Determine functional aims } \\
\text { Assessment and summary of session }\end{array}$ \\
\hline $\begin{array}{l}\text { 5th session } \\
\text { Assertive training }\end{array}$ & $\begin{array}{l}\text { Learn assertive techniques to deal with criticism, conflicts, and expressive self to prevent addictive behaviors } \\
\text { Apply these strategies } \\
\text { Determine functional aims } \\
\text { Assessment and summary of session }\end{array}$ \\
\hline $\begin{array}{l}\text { 6th session } \\
\text { Communication skills }\end{array}$ & $\begin{array}{l}\text { Concept of the importance of face to face communication in in social aspects } \\
\text { Gain skills in effective listening and dialog } \\
\text { Develop skills to communicate effectively with friends and teachers } \\
\text { Avoid social isolation and addictive behaviors } \\
\text { Assessment and summary of session }\end{array}$ \\
\hline $\begin{array}{l}\text { 7th session } \\
\text { Effective use of time }\end{array}$ & $\begin{array}{l}\text { Learn strategies for efficient use of time to prevent addictive behaviors } \\
\text { Apply these strategies } \\
\text { Determine functional aims } \\
\text { Assessment and summary of session }\end{array}$ \\
\hline $\begin{array}{l}\text { 8th session } \\
\text { Assessment }\end{array}$ & $\begin{array}{l}\text { Summary of sessions; reviewing experiences during the process } \\
\text { Members share assessment of personal development and group development during the process } \\
\text { Ensure positive emotions are felt at the end of the process } \\
\text { Final activity and appropriate finish to the group }\end{array}$ \\
\hline
\end{tabular}

\section{Study procedures}

The Internet Addiction Test (IAT) scale and Social Skills Questionnaire (SSQ-U) were uploaded to the website of An-Najah National University. Students who are interested in participation of this research were asked to answer the two measures. Candidates who received high scores on (IAT) were invited to attend a meeting in the eLearning Center at An-Najah University to inform them about the training program and conditions of participation. The total number of students who agreed to participate in this program were 30 students. Participants were randomly assigned to either wait list or treatment (15 in each condition). The experimental group received a group training program to reduce addictive Internet behaviors and improve social skills, the group intervention lasted for 2 months in summer semester of the academic year 2019, and it was started on 6 May 2019 till 24 June 2019. The performance of the two groups related to social skills and addictive behaviors was measured before and after the intervention.

\section{Data analysis}

To examine the degree of addictive Internet addiction and social skills among university students, means and standard deviations were used. Moreover, two-way analysis of covariance (ANCOVA) test was used to test the differences in addictive Internet behaviors and social skills on pre- and post-tests due to study variables: treatment and gender. Analysis of covariance (ANCOVA) is a statistical technique that blends analysis of variance and linear regression analysis. It is a more sophisticated method of testing the significance of differences among group means because it adjusts scores on the dependent variable to remove the effect of confounding variables. ANCOVA is based on inclusion of additional variables (known as covariates) into the model that may be influencing scores on the dependent variable. ANCOVA is 
an appropriate test for experimental studies, as it control the effect of pretest and calculate the differences between experimental and control groups on posttest (Mandel 2012).

\section{Ethics}

The research was conducted in line with the ethical guidelines of the American Psychological Association (APA, 2010) and the Declaration of Helsinki (2013) and had been approved by the An-Najah National University IRB (Protocol number 11 Jun). Informed consent was obtained before data were collected from the participants.

\section{Findings}

Means and standard deviations were calculated for study variables (group and gender) on pre- and post-tests for addictive behaviors and social skills as shown in Table 2 and Fig. 1.

Results of Table 2 show apparent differences in means of addictive behaviors and social skills according to study variables, to test the significance of these differences; ANCOVA test was calculated as shown in Tables 3 and 4.

Results of Table 3 show significant differences between experimental and control group in addictive behaviors in favor of experimental group, whereas there are no significant differences in addictive behaviors between males and females.

Results of Table 4 show significant differences between experimental and control group in social skills in favor of experimental group, whereas there are no significant differences in social skills between males and females.

\section{Discussion}

The current study was conducted to examine the efficacy of a specific training program aimed at increasing

Table 2 Means and standard deviations for study variable on pre- and post-tests

\begin{tabular}{|c|c|c|c|c|c|c|}
\hline \multirow{2}{*}{$\begin{array}{l}\text { Dependent } \\
\text { variable }\end{array}$} & \multirow[t]{2}{*}{ Variables } & \multicolumn{3}{|c|}{ Pre-test } & \multicolumn{2}{|c|}{ Post-test } \\
\hline & & No. & $M$ & S.D & $\bar{M}$ & S.D \\
\hline \multirow[t]{5}{*}{ Addictive behaviors } & Experimental & 15 & 3.87 & 0.26 & 2.83 & 0.15 \\
\hline & Control & 15 & 3.89 & 0.12 & 3.88 & 0.16 \\
\hline & Male & 14 & 3.86 & 0.13 & 3.27 & 0.55 \\
\hline & Female & 16 & 3.89 & 0.25 & 3.44 & 0.51 \\
\hline & Total & 30 & 3.87 & 0.17 & 3.35 & 0.24 \\
\hline \multirow[t]{5}{*}{ Social skills } & Experimental & 15 & 2.85 & 0.26 & 3.75 & 0.28 \\
\hline & Control & 15 & 2.92 & 0.19 & 2.93 & 0.21 \\
\hline & Male & 14 & 2.87 & 0.16 & 3.42 & 0.46 \\
\hline & Female & 16 & 2.90 & 0.28 & 3.27 & 0.52 \\
\hline & Total & 30 & 2.88 & 0.25 & 3.34 & 0.24 \\
\hline
\end{tabular}

social skills and decreasing addictive behaviors related to Internet addiction on the actual social skills and Internet use of university students in the West Bank of Palestine. The initial results indicate that in fact both social skills and problematic Internet usage behaviors were positively affected by the training program, with no difference related to gender in the target population.

Given the high vulnerability and critical developmental stage of the target population (university age individuals), the potential positive effects of a treatment that addresses both social skills and excessive Internet use could provide a critical intervention which would increase the overall coping skills of individuals preparing for adulthood and professional responsibilities as well as assisting them in avoiding or remediating addictive problematic behaviors not only in Internet use but other potential addictive behaviors including smoking, eating, and emotional management which could positively influence their lives and future families.

Magalhães et al. (2020) noted the importance of intervention programs based on mindfulness and social skills to increase resistance to peer pressure. In their study training students in social skills decreased the effect of peer pressure to use tobacco and alcohol. Programs based on increasing mindfulness skills and compassion may also promote general mental health outcomes and emotional resilience which may protect youth against a wider range of maladaptive and addictive behaviors.

The findings revealed that improving social skills among university students was associated with a decrease in problematic Internet use only when participants in the intervention group when they met the following these conditions: they perceived an improvement in their social skills, they learned alternative ways to communicate with others, and they felt more effective in regard to problem solving strategies. The perceived change in social effectiveness might have been the critical change agent in decreasing Internet use.

Treatment programs utilizing a variety of core skills have been examined in multi-cultural populations as well. In 2019, Balci and Uysal conducted a study for primary school aged children in Turkey directed specifically at healthy Internet use by school nurses. Treatment included both sessions for the children aimed at increasing real life social skills and included two sessions aimed at teaching parents how to manage their children's use of the Internet. The study concluded that children and parents exposed to treatment made significant decreases in use.

Yang and Kim (2018) looked at the concept of selfefficacy in middle school children and its relationship to addictive pattern Internet use related to a treatment protocol applied by school nurses which targeted selfefficacy and self-regulation. This study verified the 


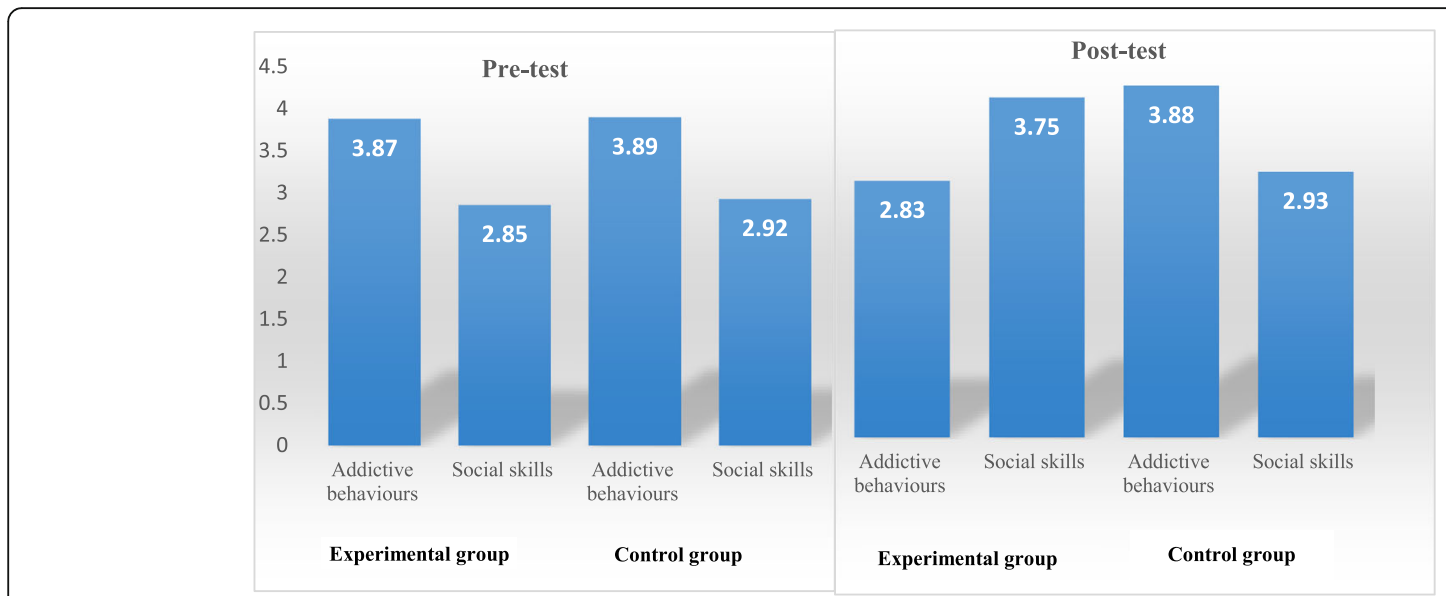

Fig. 1 Addictive behaviors and social skills on pre- and post-tests for study groups

negative dependent relationship of self-efficacy which when it increased due to treatment resulted in a decrease in addictive behavior patterns related to Internet use. Abolfathi et al. (2014) also worked with middle school students in Tehran targeting communication skills. The author found that treatment both augmented communication skills and resulted in less addictive level usage of the Internet in his sample.

Fang et al. (2011) focused on university students in China offering an on-line course directed at specific strategies to reduce excessive and addictive pattern Internet use. The study found that even with having all treatment sessions on line, their treatment was able to reduce usage in this highly vulnerable group of young adults.

Wölfling et al. (2014) used a treatment protocol of traditional Cognitive Behavioral Therapy (CBT) with adult males already exhibiting addictive patterns of Internet use. They found that not only did they have an unprecedented $70 \%$ completion rate for treatment but also that results included both a drop in addictive behaviors related to Internet usage and a reduction in general psychology symptoms including depression and anxiety.

The study of course is not without internal limitations. The sample is small and highly self-selected as it recruited only individuals who self-reported as having both excessive use and the desire to participate. It is unclear what the results would be in a more diverse less selfmotivated population. The population is also highly restricted in terms of geography, life-style, age, and education. All of the samples are university students, under the age of 30, residing in Palestine, and having the particular experience of living in an occupied territory. A vast majority were unmarried, unemployed and living with their families. This population is of course characterized by high levels of leisure time, easy access to the Internet, high desire for socialization, and limited personal responsibility. It is unclear if the treatment model would work with older or younger age groups with their inherent differences in Internet use and socialization patterns.

One challenge is that both measures of social skills and addictive behaviors were based on computergenerated self-report. Studies demonstrate that what individuals are willing to self-report in an anonymous situation (computer-based questionnaire) may differ from actual behavior both due to the desire to positively self-represent and the inability to accurately calculate daily time usage especially when related to addictive behaviors (Garcia and Gustavson 1997). Some studies have utilized actual usage data from phones or computers to address these issues (Newton 2019). In the area of social skills, it is not clear that an individual with deficits in engaging or maintaining

Table 3 ANCOVA test for addictive behaviors due to study variables

\begin{tabular}{llllllll}
\hline Dependent variable & Source of variance & SS & DF & MS & F & P & Partial Eta Squared \\
\hline Addictive behaviors & Addictive behaviors (pre) & 0.005 & 1 & 0.005 & 0.20 & 0.65 & .024 \\
& Intervention Group & 8.02 & 1 & 80.02 & 304.44 & $0.000^{* * *}$ & .995 \\
& Gender & 0.004 & 1 & 0.004 & 0.16 & 0.68 & .193 \\
& Error & 0.68 & 26 & 0.02 & & & \\
& Total & 8.95 & 30 & & & \\
\hline
\end{tabular}


Table 4 ANCOVA test for social skills due to study variables

\begin{tabular}{llllllll}
\hline Dependent variable & Source of variance & SS & DF & MS & F & P & Partial Eta Squared \\
\hline Social Skills & Social skills (pre) & 0.88 & 1 & 0.88 & 0.24 & 0.34 & .007 \\
& Intervention group & 5.51 & 1 & 5.51 & 152.76 & $0.000^{* * *}$ & .99 .6 \\
& Gender & 0.01 & 1 & 0.01 & 0.41 & 0.52 & .098 \\
& Error & 0.93 & 26 & 0.03 & & & \\
& Total & 6.97 & 30 & & & & \\
\hline
\end{tabular}

${ }^{* * *} p \leq 0.001$

social interest for example will be able to selfevaluate. In such a case, a secondary neutral observer or family member might be a more accurate judge of socially appropriate skills and responses.

There has been little data analyzing critical aspects of training addressing Internet behaviors (Espada et al 2017). The model applied used a wide variety of both specific psycho-education related to addicted behaviors but also generalized skills (assertiveness, time management, etc.). There needs to be increased examination of the specificity of effect of each module of treatment to identify which are most effective in increasing social skills and reducing problematic behaviors.

\section{Conclusion}

One concern raised with the treatment intervention is that even group treatment is costly, inconvenient, and time consuming. Current research has utilized computer-based training programs which address the availability, time, and cost of treatment interventions. It would be very interesting to test the treatment modules as an on-line program to see of similar positive results could be achieved.

Given the wide spread excessive use and potential addictive nature of Internet use and the heightened vulnerability of university aged students it may be recommended to make some education related to Internet addiction available to all students or an on-line module required for all students. This would create a community-wide scope of change and increase the efficacy of the intervention, especially for those unwilling or unable to self-identify as having problematic use.

The field of Internet addiction and intervention is a new and exciting area where there is much opportunity for growth and development. Specialized treatment groups for different age groups of users, the ability to separate productive use vs excessive use, and the role that social skills and other psychological factors play are all area for further exploration and examination.
Authors' contributions

All authors designed and conceptualized the study, collected and analyzed the data, and drafted the manuscript. All authors revised the manuscript critically for important intellectual content and approved the final version of the manuscript.

Funding

No funding was received for this study.

\section{Availability of data and materials}

The datasets used and/or analyzed during the current study are available from the corresponding author upon reasonable request.

\section{Declarations}

Ethics approval and consent to participate

The research was conducted in line with the ethical guidelines of the American Psychological Association (APA, 2010) and the Declaration of Helsinki (2013) and had been approved by the An-Najah National University IRB (Protocol number 11 Jun). Informed consent was obtained before data were collected from the participants.

\section{Competing interests}

The authors declare that they have no competing interests.

\section{Author details}

${ }^{1}$ An-Najah National University, Nablus, Palestine. ${ }^{2}$ Nationalities Service Center, Philadelphia, PA, USA.

Received: 10 November 2020 Accepted: 14 June 2021

Published online: 26 June 2021

\section{References}

Abolfathi, R., Kalantarkousheh, S., Rasouli, M., \& Nouri, N. (2014). Effectiveness of communication skills in decreasing addiction tendencies among male students from cities within Tehran province. European Journal of Experimental Biology, 4(1), 64-70

Balci, S., \& Uysal, G. (2018). Evaluation of a school-based program for Internet addiction of adolescents in Turkey. Journal of Addictions Nursing, 29(4), 43-49. https://doi.org/10.1097/JAN.0000000000000211.

Bambaeeroo, F., \& Shokpour, N. (2017). The impact of the teachers' non-verbal communication on success in teaching. J Adv Med Educ Prof, 5(2), 51-59.

Berte, D. Z., Mahamid, F. A., \& Affouneh, S. (2021). Internet addiction and perceived self-efficacy among university students. International Journal of Mental Health and Addiction, 19(1), 162-176. https://doi.org/10.1007/s11469019-00160-8.

Blachnie, A., Czuzcwar, S., \& Przepiorka, A. (2017). Type D personality, stress coping strategies and self-efficacy as predictors of Facebook intrusion. Psychiatry Research, 253, 33-37. https://doi.org/10.1016/.jpsychres.2017.03.022.

Blakemore, S. J. (2008). The social brain in adolescence. Nature Reviews Neuroscience, 9(4), 267-277 https://doi.org/10.1038/nrn2353.

Botvin, G., \& Griffin, K. (2005). Prevention science, drug abuse prevention, and life skills training: comments on the state of the science. Journal of Experimental Criminology, 1(1), 63-78. https://doi.org/10.1007/s11292-004-6462-y.

Celik, C. (2016). Educational intervention for reducing Internet addiction tendencies. The Turkish Journal on Addictions, 3(3), 375-386. https://doi.org/1 0.15805/addicta.2016.3.0021 
Champion, K., Newton, N., Stapinski, L., \& Teesson, M. (2016). Effectiveness of a universal internet-based prevention program for ecstasy and new psychoactive substances: A cluster randomized controlled trial. Addiction Research Report, 11, 1396-1405. https://doi.org/10.1111/add.1334.

Chang, Y., Chen, Y., Chou, W., Huang, M., Hu, H., \& Yen, C. (2017). Social skills deficits and their association with Internet addiction and activities in adolescents with attention-deficit/hyperactivity disorder. Journal of Behavioral Addictions, 6(1), 42-50. https://doi.org/10.1556/2006.6.2017.005.

Cheng, C., Chen, Z., \& Poon, K. (2017). Deficits in recognizing disgust facial expressions and internet addiction: Perceived stress as a mediator. Psychiatry research, 254, 211-217. https://doi.org/10.1016/j.psychres.2017.04.057.

Chiu, S. (2014). The relationship between life stress and smartphone addiction on Taiwanese university student: A mediation model of learning self-Efficacy and social self-Efficacy. Computers in Human Behavior, 34, 49-57. https://doi. org/10.1016/j.chb.2014.01.024.

Cully, J. A., \& Teten, A. L. (2008). A therapist's guide to brief cognitive behavioral therapy. Houston: Department of Veterans Affairs South Central MIRECC.

Du, Y., Jiang, W., \& Vance, A. (2010). Longer term effect of randomized, controlled group cognitive behavioural therapy for Internet addiction in adolescent students in Shanghai. Australian \& New Zealand Journal of Psychiatry, 44(2), 129-134. https://doi.org/10.3109/00048670903282725.

Espada, J., Orgilés, M., Piqueras, J., \& Torrente (2014). Association of Internet addiction with social anxiety and lack of social skills in Spanish adolescents. Terapia PsicolÓgica, 32(3), 175-184.

Facebook. (2020). Retrieved December 2018, from https://newsroom.fb.com/ company-info/

Fang, X., Miller, J., Su, W., \& Wang, Y. (2011). Internet-based intervention for the treatment of online addiction for college students in China: A pilot study of the Healthy Online Self-Helping Center. Cyber psychology, behavior, and social networking, 14(9), 497-503. https://doi.org/10.1089/cyber.2010.0167.

Fang, X., Lan, J., Liu, C., Liu, Q., Yan, N., Yuan, X., \& Zhou, Z. (2015). Multi-family group therapy for adolescent Internet addiction: Exploring the underlying mechanisms. Addictive Behaviors, 42, 1-8. https:/doi.org/10.1016/j.addbeh.2014.10.021.

Garcia, G. \& Gustavson, A.R. (1997). The science of self-report. The Observer, 1/3/ 1997. Retrieved from https//www.aps.org on 3/1/2019

Giedd, J. (2012). The digital revolution and adolescent brain evolution. Journal of Adolescent Health, 51(2), 101-105. https://doi.org/10.1016/j.jadohealth.2012.06.002.

Greco, M., Olza, F., Medrano, L., \& Prette, Z. (2009). Argentinean adaptation of the social skills inventory IHS-Del-Prette. The Spanish Journal of Psychology, 12(2), 756-766. https://doi.org/10.1017/S1138741600002122.

Halasy, K., Lindenberg, K., \& Schoenmaekers, S. (2017). A randomized efficacy trial of a cognitive-behavioral group intervention to prevent Internet Use Disorder onset in adolescents: The PROTECT study protocol. Contemporary Clinical Trials Communications, 6, 64-71. https://doi.org/10.1016/j.conctc.2017.02.011.

Hawi, N. S., \& Samaha, M. (2016). To excel or not to excel: Strong evidence on the adverse effect of smartphone addiction on academic performance. Computers \& Education, 98, 81-89. https://doi.org/10.1016/j.compedu.2016.03.007.

Hawi, S., \& Samaha, M. (2017). The relations among social media addiction, self esteem, and life satisfaction in university students. Social Science Computer Review, 35(5), 576-586. https://doi.org/10.1177/0894439316660340.

Instagram. (2020). Retrieved February 2019, from https://instagram-press.com/ourstory/

Kabasakal, Z. (2015). Life satisfaction and family functions as-predictors of problematic internet use in university students. Computers in Human Behavior, 53, 294-304. https://doi.org/10.1016/j.chb.2015.07.019.

Khazaei, F., Khazaei, O., \& Ghanbari-H., B. (2017). Positive psychology interventions for internet addiction treatment. Computers in Human Behavior, 72, 304-311. https://doi.org/10.1016/j.chb.2017.02.065

Knapp, M. L., Hall, J. A., \& Horgan, T. G. (2013). Nonverbal communication in human interaction. Cengage Learning. ISO 690

Kuss, D., Griffiths, M., Karila, L., \& Billieux, J. (2014). Internet addiction: A systematic review of epidemiological research for the last decade. Current Pharmaceutical Design, 20(25), 4026-4052. https://doi.org/10.2174/1381612 8113199990617.

Lampe, C., Ellison, N., \& Steinfield, C. (2008). Social capital, self-esteem, and use of online social network sites: A longitudinal analysis. Journal of Applied Developmental Psychology, 29(6), 434-445. https://doi.org/10.1016/j.appdev.2 008.07.002.

Lau, J., Chan, S., Chan, V., \& Mo, P. (2018). The role of social support on emotions dysregulations and internet addiction among Chinese adolescents. A structural equation model, 82, 86-93. https://doi.org/10.1016/j.addbeh.2018.01.027.
Lepp, A., Barkley, J. E., \& Karpinski, A. C. (2014). The relationship between cell phone use, academic performance, anxiety, and satisfaction with life in college students. Computers in Human Behavior, 31, 343-350. https://doi. org/10.1016/j.chb.2013.10.049.

Linkedln. (2018).Retrieved December 2018, from https://www.omnicoreagency. com/linkedin-statistics/

Liu, J., \& Wang, Y. (2017). Effects of group counseling programs, cognitive behavioral therapy, and sports intervention on Internet addiction in East Asia: A systematic review and meta-analysis. Int. J. Environ. Res. Public Health, 14(1470), 2-17. https://doi.org/10.3390/ijerph14121470.

Magalhães, F., Carraça, B., \& Gaspar de matos, M. (2020). Mindfulness selfcompassion and psychological flexibility on adolescence. In T. A. Carvalho (Ed.), What to Know about Mindfulness, (pp. 119-131). New York: Nova science publishers.

Mahamid, F. A., \& Berte, D. Z. (2019). Social media addiction in geopolitically atrisk youth. Int J Ment Health Addiction. https://doi.org/10.1007/s11469-0179870-8, 17(1), 102-111.

Mahamid, F. A., \& Berte, D. Z. (2020). Portrayals of violence and at-risk populations: symptoms of trauma in adolescents with high utilization of social media. Int J Ment Health Addiction. https://doi.org/10.1007/s11469-0189999-0, 18(4), 980-992.

Mandel, J. (2012). The statistical analysis of experimental data. Courier Corporation.

Mclaughlin, D., \& Whitty, M. (2007). On line recreation: The relationship between loneliness, internet self-efficacy and the use of internet for entertainment purposes. Computers in Human Behavior, 23(3), 1435-1446. https://doi.org/1 0.1016/j.chb.2005.05.003.

Moran, V., Olaz, F., \& Prette, Z. (2015). Social Skills Questionnaire for Argentinean College Students (SSQ-U) Development and Validation. The Spanish Journal of Psychology, 18(95), 1-11. https://doi.org/10.1017/s.jp.2015.92.

Mourtada, R. \& Salem, F. (2015). Civil Movements in the Arab World - The Impact of Facebook and Twitter. Arab Social Media Report. Retrieved from file:///C:/ Users/XBYW1/Downloads/ASMR2_En_Final.pdf

Newton, C. (2019) Why screen-time can't measure the effect of smartphones on our wellbeing. Retrieved from https//www.theverge on 3/1/2019.

Satici, S. A., \& Uysal, R. (2015). Well-being and problematic Facebook use. Computers in Human Behavior, 49, 185-190. https://doi.org/10.1016/j.chb.201 5.03 .005

Sherman, L. E., Michikyan, M., \& Greenfield, P. M. (2013). The effects of text, audio, video, and in-person communication on bonding between friends. Cyberpsychology: Journal of Psychosocial Research on Cyberspace, 7(2). https:// doi.org/10.5817/CP2013-2-3.

Spencer, N. (2006). Social equalization in youth: evidence from a cross-sectional British survey. European Journal of Public Health, 16(4), 368-375. https://doi. org/10.1093/eurpub/cki222.

Sriwilai, K., \& Charoensukmongkol, P. (2015). Face it, don't Facebook it: Impacts of social media addiction on mindfulness, coping strategies and the consequence on emotional exhaustion. Stress and Health, 32(4), 427-434 https://doi.org/10.1002/smi.2637.

Twitter. (2020). Retrieved October 2018, from https://www.omnicoreagency.com/ twitter-statistics/

Wölfling, K., Beutel, M. E., Dreier, M., \& Müller, K. W. (2014). Treatment Outcomes in Patients with Internet Addiction: A Clinical Pilot Study on the Effects of a Cognitive-Behavioral Therapy Program. BioMed Research International, 2014, 1-8. https://doi.org/10.1155/2014/425924

Wolfing, K., Müller, K. W., Giralt, S., \& Beutel, M. E. (2011). Negative affectivity and deficient stress coping in persons with Internet addiction. Suchttherapie, 57(1), 27-37. https://doi.org/10.1024/0939-5911.a000081.

Yang, S.-Y., \& Kim, H.-S. (2018). Effects of a prevention program for internet addiction among middle school students in South Korea. Public Health Nursing, 35(3), 246-255. https://doi.org/10.1111/phn.12394

Yao, M., \& Zhong, Z. (2014). Loneliness, social contacts and Internet addiction: A cross-lagged panel study. Computers in Human Behaviour, 30, 164-170. https://doi.org/10.1016/j.chb.2013.08.007.

Zarb, J. M. (2007). Developmental cognitive behavioral therapy with adults. New York: Taylor \& Francis.

\section{Publisher's Note}

Springer Nature remains neutral with regard to jurisdictional claims in published maps and institutional affiliations. 